\title{
BLOCK ORTHORECTIFICATION AND MOSAIC OF 1960s DISP IMAGES IN QUJING, YUNNAN
}

\author{
Xinyue Peng ${ }^{1}$, Guoqing Zhou ${ }^{1}$, , Hongbo Yan ${ }^{1}$, Tao Yue ${ }^{1}, \mathrm{Jie} \mathrm{Liu}^{2}$, Yexuan $\mathrm{Mu}^{1}$ \\ ${ }^{1}$ Guangxi Key Laboratory of Spatial Information and Geomatics, Guilin University of Technology, No. 12 Jian'gan Road, Guilin, \\ Guangxi 541004, China \\ ${ }^{2}$ College of Earth Science, Guilin University of Technology, No. 12 Jian'gan Road, Guilin, Guangxi,541004, China
}

KEY WORDS: DISP, Orthorectification, Mosaic, Color balance, Rocky desertification, Karst

\begin{abstract}
:
Remote sensing digital image mosaic refers to the splicing of two or more remote sensing images into a panoramic image to meet the application requirements of wide images. It is becoming more and more important to cover large areas of mosaic images. The data pre-processing and mosaic process are different for different images. In this paper, the declassified intelligence satellite photography (DISP) in the 1960s was used as experimental data, taking Qujing in Yunnan Province as an example, a total of 92 scene images were used for DISP image processing and seamless mosaic. Firstly, In order to make full use of the image, it is necessary to perform orthorectification on the KH-4B images covering Qujing. Considering that the satellite orbit parameters and other parameters are unknown, several models for orthorectification of satellite images are proposed. After experiments and analysis, it is concluded that the third order polynomial is the optimal. Secondly, given the problem of radiation imbalance caused by the difference of time phase, the histogram equalization method is used for color balance, the image of the seam line is eliminated. Finally, the seamless mosaic is achieved. The map of the 1960s DISP in Qujing is obtained. The map can provide material basis for future research and other fields. The processing methods of historical images can be used for reference by others.
\end{abstract}

\section{INTRODUCTION}

Historical remote sensing images have been of great research significance and application value in the fields of ecological environment change, sustainable development, agricultural development, urban expansion, coastline change, ice dynamic monitoring and so on(Mi et al.,2014). Rocky desertification in Karst area is one of the most serious natural disasters in three provinces of southwest China. The emergence of rocky desertification restricts the development of local economy and aggravates poverty. Therefore, in order to grasp the law of time and space development of rocky desertification, it is necessary to carry out the overall mosaic in the early karst areas. Taking Qujing in Yunnan Province as an example, this paper makes a thematic mapping of Qujing Karst geomorphology in the early 1960 s. Since the late 1990s, the problem of rock desertification has attracted great attention of domestic academic circles and national governments. In many studies, most scholars use TM images in the 1990s to get Karst maps. Early studies have mostly been involved in the late $1990 \mathrm{~s}$, rather than using historical images. The US Geological Survey, after obtaining permission, published Declassified intelligence satellite photography (DISP) images from the 1960s, some of which covered most of China. Zhou et al., (2002a, 2002b, and 2003) used images to depict ice sheet in Greenland in the 1960s and analyzed the changes of ice sheet. Mi et al., (2014) used a partitioned third order polynomial model to correct the DISP image with unknown orbital parameter information. Qiao et al., (2018) used the DISP image to analyze the changes in the coastline of Shanghai. This paper uses the DISP image to carry out the seamless mosaic of the Qujing karst land in Yunnan, which has important historical significance.
However, to make full use of DISP images, difficulties in mapping a map using DISP images are as follows:

(1) The parameters of the DISP image satellite are unknown, so it is difficult to find the appropriate orthorectification model to satisfy the rectification of large amount of data.

(2) For modern high resolution images, the change of ground objects makes it difficult to find control points due to age, the quantity is insufficient and the quality is not high.

(3) The single scene image belongs to long strip shape, the longitude span is large, and the curvature of the earth has a great influence.

(4) There are a large number of clouds in some images.

\section{DATA}

\subsection{Study Area}

Qujing is located in the eastern part of Yunnan Province (see Figure 1), at the junction of Yunnan, Guizhou and Guangxi provinces. It is located between $102^{\circ} 42^{\prime}-104^{\circ} 50^{\prime}$ east longitude and $24^{\circ} 19^{\prime}-27^{\circ} 03^{\prime}$ north latitude, with a total area of $32,565 \mathrm{~km}^{2}$.

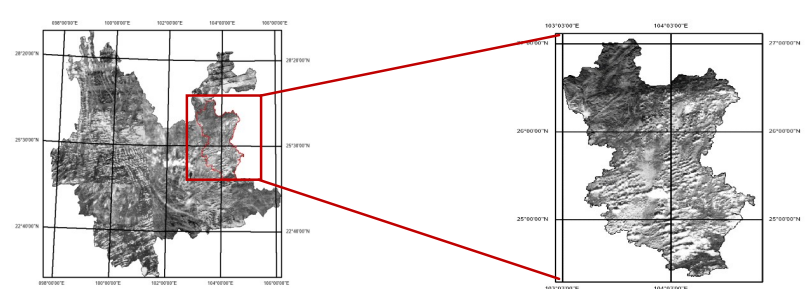

Figure 1. Study area

* Corresponding author: Guoqing Zhou; E-mail: gzhou@glut.edu.cn 
Qujing is the second most serious city of rocky desertification in Yunnan Province. The area of rocky desertification is about $4445 \mathrm{~km}^{2}$, accounting for $31.3 \%$ of the karst area (end of 2005). It has a typical Karst geomorphology.

\subsection{Declassified Intelligence satellite photography (DISP)}

Declassified intelligence satellite photography is a military image taken by the first generation of American photography reconnaissance satellites, including the system code names CORONA, ARGON, and LANYARD. On February 24, 1995, after an executive order signed by US President Bill Clinton, the DISP image was made public. More than 860,000 surface images collected from 1960 to 1972 were published. Only the National Archives and Records Administration (NARA) and the United States Geological Survey (USGS) provide and sell DISP products. The DISP image used in this article is from the USGS website (http://earthexplorer.usgs.gov/), some of the free DISP images can be found and downloaded through Earth Explorer's interactive query system, while some need to be purchased. These historical images provided strong support for US military development and national security in the early days, as well as effective research assistance for environmentalists, scientists, scholars, and historians. Zhou et al., (2003) performed orthorectification and mosaic processing on 1962 and 1963 ARGON images covering Greenland. K.KIM et al., (2006) also used the 1963 ARGON satellite imagery to conduct an environmental change study in Antarctica in 2006. The images used in both applications belong to DISP images.

In this paper, DISP images (see Figure 2) are used for orthorectification and mosaic of Qujing Karst area. A total of 23 scene images, including Missions 1002-2 in December 1967, Missions 1105-2 in November 1968. The data was purchased from the USGS official website. CORONA belongs to the KeyHole 4 (KH-1-4) series. The satellite images of the two Missions belong to the $\mathrm{KH}-4 \mathrm{~B}$ series, the resolution is $1.8 \mathrm{~m}$, the imaging width is $13.8188 \mathrm{~km}^{2}$, and the satellite height is $150 \mathrm{~km}$

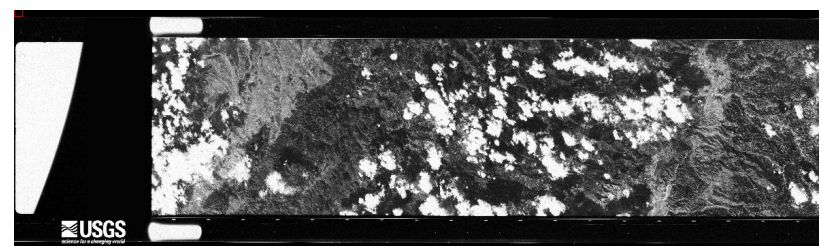

Figure 2. DISP original image

\section{ORTHORECTIFICATION EVALUATION.}

\subsection{Imagery Rectification}

Orthorectification refers to the process of error elimination or correction of the acquired geometric distortion of the remote sensing image relative to the real surface target (Sun, 2003). The DISP image conforms to the deformation of the panoramic camera (Sohn et al., 2002). Like other optical images, DISP geometric deformation include factors such as sensor imaging, external orientation elements, atmospheric refraction and earth curvature, topographic fluctuation, etc. Orthorectification includes rough rectification and fine rectification. Orthorectification using ground control points (GCPs) is fine rectification. This paper uses fine rectification, including pixel coordinate transforming and pixel brightness value resampling. The premise of image orthorectification is to establish an effective mathematical correction model. So far, there are many types of correction models. For different imaging products, the most suitable correction model for the image should be selected. Zhou et al., (2002) combined the bundle adjustment method with the satellite orbital parameter to perform orthorectification on the DISP image. Kim (2004) used DEM data to perform bundle triangulation and orthorectification for DISP images. Mi et al., (2014) adopted a block polynomial method to geometric positioning the DISP in Shanghai. Zhou, et al., (2016) proposed second-order polynomial equation-based block adjustment for orthorectification of DISP imagery.

Considering the characteristics of the DISP image, such as the internal and external orientation elements and camera lens distortion, the parameters of the satellite are unknown. Therefore, the correction method that requires satellite parameters, such as the rational function model and the collinear equation, cannot be used when correcting the DISP image. This paper selects Rubber Sheeting and polynomial correction model to correct the KH-4B images.

Rubber Sheeting geocentric correction model expressed:

$$
\begin{aligned}
& X=a x_{1} y_{1}+b x_{1}+c y_{1}+d \\
& Y=e x_{1} y_{1}+f x_{1}+g y_{1}+h
\end{aligned}
$$

Where $(X, Y)$ represents the coordinates to be calculated for the second reference system, $\left(x_{1}, y_{1}\right)$ are given coordinates in the first reference system;

Polynomial orthorectification model expressed:

$$
\begin{aligned}
& x=a_{0}+\left(a_{1} X_{G}+a_{2} Y_{G}\right)+\left(a_{3} X_{G}{ }^{2}+a_{4} X_{G} Y_{G}+a_{5} Y_{G}{ }^{2}\right)+\ldots \\
& y=b_{0}+\left(b_{1} X_{G}+b_{2} Y_{G}\right)+\left(b_{3} X_{G}{ }^{2}+b_{4} X_{G} Y_{G}+b_{5} Y_{G}{ }^{2}\right)+\ldots
\end{aligned}
$$

Where $\left(X_{G}, Y_{G}\right)$ is the ground point coordinates, $(x, y)$ is the image point coordinates of the imagery;

The polynomial correction model avoids the spatial geometric model of the imaging and directly simulates the image deformation itself. Rubber Sheeting is a process that simulates image deformation by assuming that image distortion is the result of higher-order translation, scaling, rotation, affine, bending, and deformation. In addition, considering the large amount of experimental data cloud, the local precision is low when the single scene image is corrected. For this reason, the whole scene image is segmented according to the area where the cloud layer is located, as shown in Figure 3. Taking DS11052264DF039 as an example, the whole scene image is divided into four parts according to the area where the cloud layer is located. In the area where the cloud layer is mostly, the boundary has a clear landform and a certain overlapping area exists between adjacent parts.

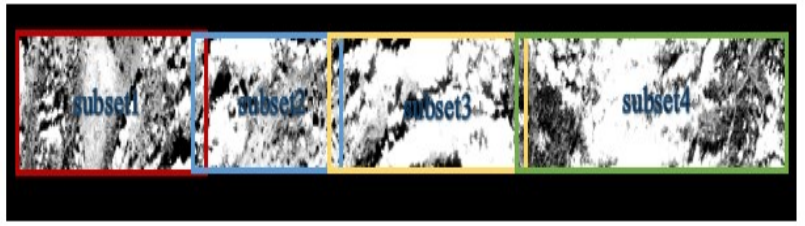

Figure 3. Block processing of DISP images (DS11052264DF039)

\subsection{Selection of GCPs}

In the orthorectification process, the correction precision depends on the selection of GCPs, and the coefficients of the correction model are calculated by the coordinates of the 
selected GCPs. The GCPs should be distributed as evenly as possible throughout the entire area of the image, and the edges must have a sufficient number of control points. Since the image selected in this paper is in the 1960s, it is difficult to find reference images or maps of the same era, so there are some problems in the selection of ground control points. In this paper, the Google Earth image is used to select the GCPs of the image in the 1960s. However, due to the age, there are still great obstacles in the selection of GCPs. There are three aspects:

(1) In mountainous and desert areas, there are no obvious feature points.

(2) Part of the image has a large amount of cloud cover, obscuring the surface and not recognizing.

(3) Due to the construction of the city, the actual ground objects have undergone great changes.

In order to improve the precision, it is necessary to select GCPs on the obvious objects, such as road bends, river intersections, lake corners, etc. (see Figure 4). GCPs must be evenly distributed to avoid concentration and distortion of specific areas. In order to get better correction effect, the rubber sheeting, second order polynomial and third order polynomial model are respectively selected to correct the image. Figure 5 shows the GCPs distribution in subset 1 , which includes 50 GCPs and 10 check points. The 10 check points are evenly distributed over the subset1 and used to calculate the precision of the correction results. Table 1 shows the accuracy of the correction results.

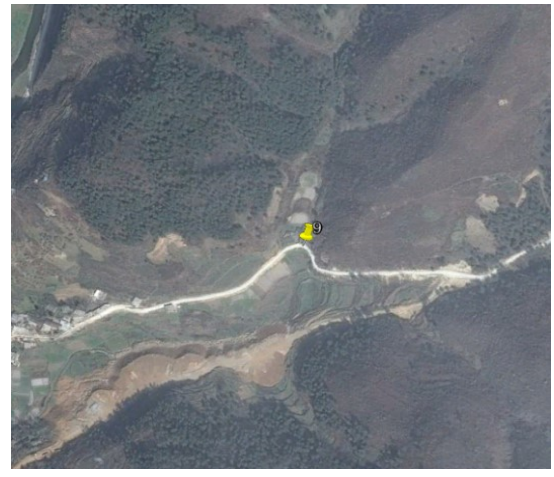

(a) Google Earth map

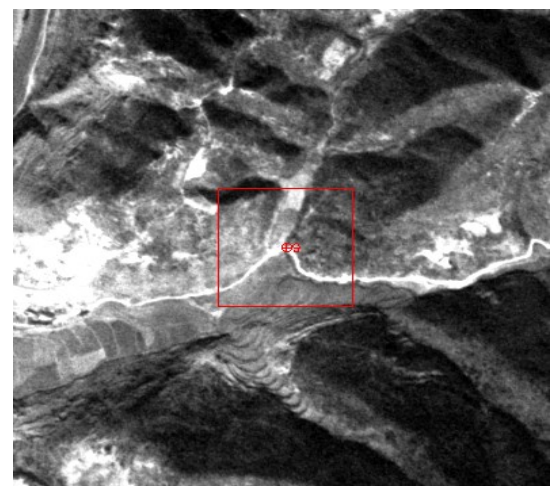

(b) DISP image

Figure 4. Corresponding to the selection of Google Earth map images

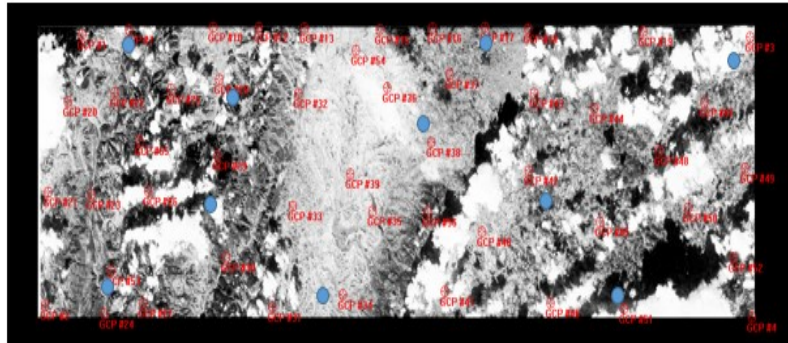

Figure 5. GCPs selection and check points (blue is check point)

\subsection{Result Analysis}

The data from Table 1 and Table 2 show that the correction accuracy of the polynomial and Rubber Sheeting is not much different. Among them, the Rubber Sheeting correction model has the smallest RMS. In theory, Rubber Sheeting has the highest correction precision and the best correction effect. However, in the actual experiment, rubber sheeting has the phenomenon of excessive edge distortion. The reason is that the Rubber Sheeting correction model only stretches the image within the range of GCPs, so that the undistributed GCPs edge part of the image is overdistorted after stretching. The third order polynomial has an RMS of 25.31, and at the same time it can correct the global image, so it is concluded that the third polynomial correction model is the best. Since the Google Earth image is only used when correcting the image, the correction precision cannot reach the single digit level. However, by contrasting with the TM image, the images can completely coincide, so the correction results meet the requirements.

Table 1. Accuracy comparison of orthorectification models

\begin{tabular}{lccc}
\hline & $\begin{array}{c}\text { Rubber } \\
\text { Sheeting }\end{array}$ & $\begin{array}{c}\text { Second Order } \\
\text { Polynomial }\end{array}$ & $\begin{array}{c}\text { Third Order } \\
\text { Polynomial }\end{array}$ \\
\hline RMS $(\mathrm{m})$ & 22.95 & 26.47 & 25.31 \\
\hline
\end{tabular}

Table 2. Accuracy comparison of check points

\begin{tabular}{cccc}
\hline & X Residual(m) & Y Residual(m) & RMS(m) \\
\hline $\begin{array}{c}\text { Rubber } \\
\text { Sheeting }\end{array}$ & 8.0242 & 6.4794 & 10.31 \\
$\begin{array}{c}\text { Second } \\
\text { Order } \\
\text { Polynomial }\end{array}$ & 9.7064 & 8.7608 & 13.08 \\
$\begin{array}{c}\text { Third } \\
\text { Order } \\
\text { Polynomial }\end{array}$ & 11.1917 & 6.4802 & 12.93 \\
\hline
\end{tabular}

\section{MOSAIC PRODUCT}

\subsection{Single scene image Mosaic}

Remote sensing digital image mosaic refers to the splicing of two or more remote sensing images into a panoramic image to meet the application requirements of wide images (wen, 2009). In this paper, a method based on overlapping image is adopted to solve the problem of remote sensing image mosaicking, smoothed overlapping area and realized DISP seamless mosaicking. 
After the orthorectification, the subset 1-4 are used to mosaic a single scene image, as shown in Figure 6, and then the mosaicking of the adjacent images is performed. The Mosaic process is shown in Figure 6, 7.

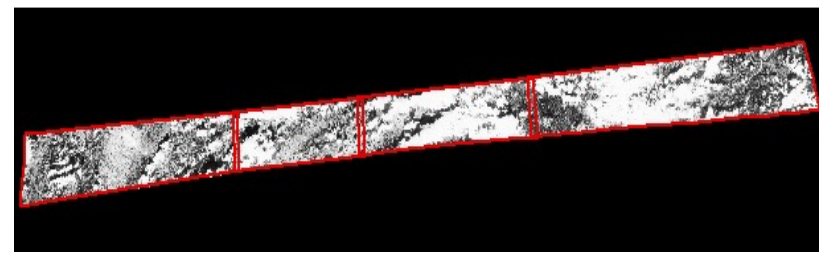

Figure 6. Subset 1-4 mosaicking of DS1105-2264DF039

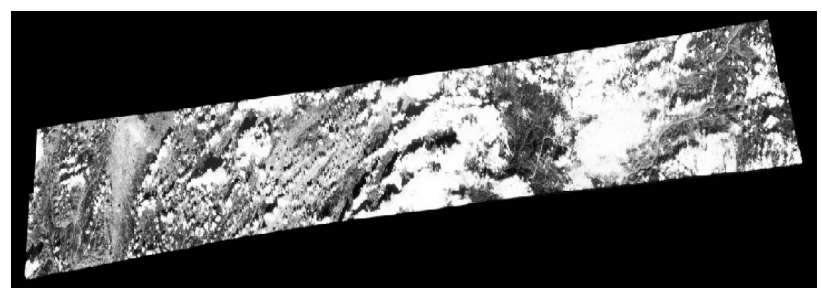

Figure 7. Double scene image mosaicking of DS11052264DF039 and DS1105-2264DF039

\subsection{Radiation Balance}

For remote sensing images obtained from different sensors, with different time and different weather conditions, the same features in the overlapping area will produce a difference in color during the mosaic process, which is manifested as radiation inconsistency. This situation leads to different color tones after the mosaic, and the seam line is obvious, which affects the beauty and quality of the overall image and reduces the application effect. The DISP images selected in this paper come from two different time periods, the orbit is different, and there is a large difference in color between adjacent images. Chen et al., (2014) used a parallel relative radiation normalization method to eliminate the influence of seam lines. $\mathrm{Yu}$ et al., (2017) proposed an adaptive global to local color equalization method. In this paper, the histogram equalization method is used to perform radiation balance processing on the images, and the seam line is selected by using the weight, and the mosaic images is smoothed and feathered to reduce the difference in color.

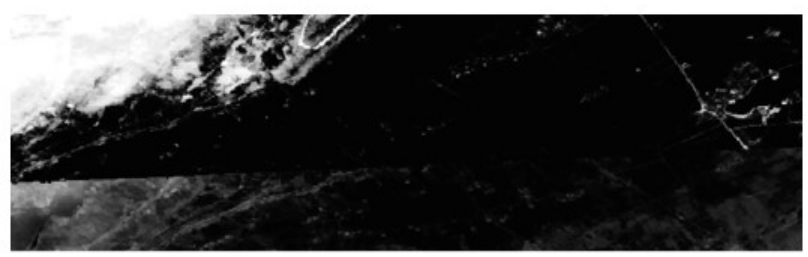

(a)

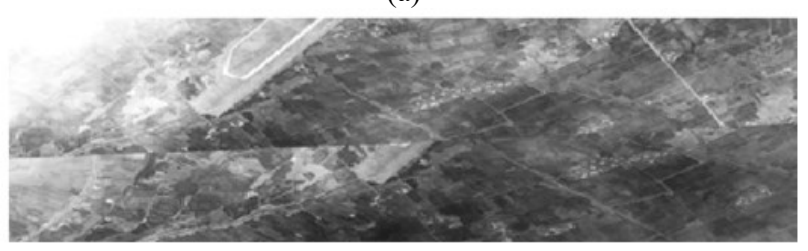

(b)

Figure 8. Radiation balance ((a) is before radiation balance, and (b) is after radiation balance)

\subsection{Mosaicking Result}

In this paper, the order of correction first and mosaic is adopted, and the four parts belonging to the same scene image are connected, then the adjacent two images are connected in pairs, and then the whole mosaic is carried out. This order helps to eliminate image distortion and improve the quality of the mosaic. Finally, through the overall mosaic of the two images, a total of 92 images, and in the mosaic process, the histogram equalization method is used for the adjacent overlapping regions, and the nearest neighbor pixel method is selected for image resampling, and smoothing and feathering are performed simultaneously. After these, the first map of Qujing in Yunnan Province in the 1960s, as shown in Figure 9. The map provides the basis for future research.

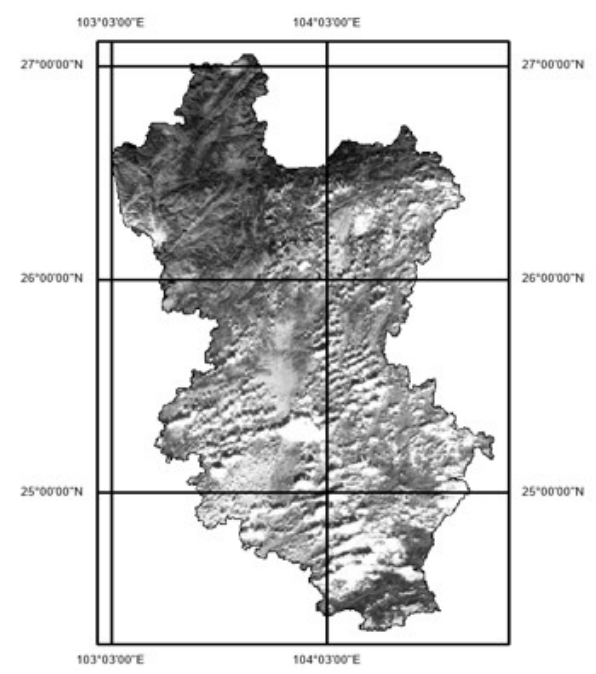

Figure 9. Qujing DISP mosaic results in the 1960s

\section{CONCLUSIONS}

Remote sensing historical images can provide great help in the monitoring of time and space changes and achieve sustainable development. The earliest declassified of DISP images was in the 1960s, and some of the images covered China, which has great significance for remote sensing application research. Based on the data processing of DISP historical images, including orthorectification and radiation balance, several models for rectification, Rubber Sheeting, second polynomial and third polynomial are proposed. Finally, the third polynomial correction model is the best, and completed the high-resolution full-color image mosaic of the 1960s in Yunnan Province, and obtained the first special map of the 1960s in Qujing. In this paper, the method of selecting historical image ground control points can provide reference for others. The map can provide material basis for future research and other fields.

\section{ACKNOWLEDGEMENTS}

This paper is financially supported by the National Natural Science of China under Grant numbers 41431179, 41961065; Guangxi Innovative Development Grand Grant under the grant numbers GuikeAA18118038, GuikeAA18242048; the National Key Research and Development Program of China under Grant numbers 2016YFB0502501 and the BaGuiScholars program of Guangxi (Guoqing Zhou). 


\section{REFERENCES}

Chong C., Zhen C., et al, 2014. Parallel relative radiometric normalisation for remote sensing image mosaic. Computers and Geosciences, 73, 28-36.

Kim, K.T., 2004. Satellite Mapping and Automated Feature Extraction: Geographic Information System-Based Change Detection of the Antarctic Coast. The Ohio State University.

KIM, K., Jezek, K.C., LIU, H., 2006. Orthorectified image mosaic of Amtarctica from 1963 Argon satellite photography: image processing and glaciological applications. International Journal of Remote Sensing, 28(23), 17.

Lei Y., Chi L., et al, 2017. An auto-adapting global-to-local color balancing method for optical imagery mosaic. ISPRS Journal of Photogrammetry and Remote Sensing, 132, 1-19.

Mi, H., Qiao, G., Li, T., Qiao, S., 2014. Declassified historical satellite imagery from 1960 and geometric positioning evaluation in Shanghai, China. In: Second International Conference, GRMSE 2014. Ypsilanti, MI, USA, October 3-5. pp. 283-292.

Qiao, G., Mi, H., Wang, W .a., Tong, X. h., Li, Z. b., Tan, L., Liu, S. j., Yang H., 2018.55-year (1960-2015) spatiotemporal shoreline change analysis using historical DISP and Landsat time series data in Shanghai. International Journal of Applied Earth Observations and Geoinformation, 68.

Roger X., et al, 2010. Automatic Generation of Seamless Mosaics over Extensive Areas from High Resolution Imagery.

Sun, J. b., 2003. Principles and application of remote sensing. Wuhan University Publication, Wuhan.

Sohn, H.G., Kim, G.H., Yun, K.H., 2002. Rigorous sensor modeling of early reconnaissance CORONA imagery for monitoring urban growth. In: Geoscience and Remote Sensing Symposium. IEEE International Geoscience and Remote Sensing Symposium (IGARSS 2002)/24th Canadian Symposium on Remote Sensing. pp. 1929-1931.

U.S. Geological Survey, http: //earthexplorer.usgs.gov/.

Wen, H. y., 2009.Research on Mosaic Algorithm of Remote Sensing Image. Huazhong University of Science \& Technology, Wuhan.

Zhou G., Jezek K., 2002: Orthorectification of 1960's satellite photographs (DISP) Covering Greenland, IEEE Tran. on Geoscience and Remote Sensing, vol. 40, no. 6, pp. 1247-1259.

Zhou, G., and K. C. Jezek. 2002. Satellite photograph mosaics of Greenland from the 1960s era. International Journal of Remote Sensing, 23(6):1143-1159.

Zhou G., Jezek K., Allen T.R., 2003. Greenland ice sheet mapping using 1960s DISP imagery. IEEE International Geoscience \& Remote Sensing Symposium. IEEE.

Zhou G., et al, 2016. Second-Order Polynomial Equation-Based Block Adjustment for Orthorectification of DISP Imagery. Remote Sensing, vol. 8, no. 8, pp. 1-18. 